\title{
Selective clamping hand-assisted laparoscopic partial nephrectomy for localized renal tumors: A novel technique
}

\author{
Bum Sik Tae, Byeong Jo Jeon, Nam Cheol Kim, Hoon Choi, Jae Hyun Bae, Jae Young Park \\ Department of Urology, Korea University Ansan Hospital, Korea University College of Medicine, Ansan, Korea
}

Purpose: In this study, we described our initial experience and analyze the learning curve of segmental renal artery branch clamping with hand-assisted laparoscopic partial nephrectomy (PN) using special instruments.

Materials and Methods: We conducted a retrospective review of consecutive cases of hand-assisted laparoscopic PN (LPN) between May 2015 and April 2018. Patient demographics, tumor characteristics, perioperative details, postoperative complications, and warm ischemic time for segmental artery branch clamping were included in our analysis. We used the cumulative sum (CUSUM) method to generate learning curves.

Results: Segmental renal artery branch clamping was successfully completed in 16 of 20 patients. The median tumor size was 2.9 $\mathrm{cm}$ (range, 1.7-7.0 cm), median operation time was 185 minutes (range, 140-245 minutes), median blood loss was $291 \mathrm{~mL}$ (range, 100-600 mL), and median hospital stay was 5 days (range, 4-7 days). The median selective ischemic time was 21 minutes (range, 16-35 minutes). No patient had postoperative complications, acute or delayed bleeding. The median pre- and postoperative serum creatinine levels $(0.91$ and $0.98 \mathrm{mg} / \mathrm{dL}$, respectively), and the pre- and postoperative estimated glomerular filtration rate (89.7 and $79.6 \mathrm{~mL} / \mathrm{min}$ per $1.73 \mathrm{~m}^{2}$, respectively) were similar. Upon visual assessment of the CUSUM plots, a downward inflection point for decreasing total operation time was observed in the 9th case and estimated blood loss in the 12th case.

Conclusions: Our study shows that segmental renal artery branch clamping hand-assisted LPN for localized renal tumors is feasible, safe, and has a relatively short learning curve.

Keywords: Ischemia; Laparoscopy; Nephrectomy

This is an Open Access article distributed under the terms of the Creative Commons Attribution Non-Commercial License (http://creativecommons.org/licenses/by-nc/4.0) which permits unrestricted non-commercial use, distribution, and reproduction in any medium, provided the original work is properly cited.

\section{INTRODUCTION}

The National Comprehensive Cancer Network (NCCN) guidelines recommend partial nephrectomy $(\mathrm{PN})$ to preserve renal function in patients with a localized tumor [1]. Open PN has been the gold standard treatment for small renal masses for several decades, because of its excellent oncologic and functional outcomes [2]. Meanwhile, owing to advances in surgical techniques, laparoscopic PN (LPN) is one of the most popular minimally invasive nephron-sparing options for treating localized renal tumors [3]. In addition, in carefully selected patients, LPN provides 10-year oncologic

Received: 1 November, 2018 • Accepted: 8 January, 2019

Corresponding Author: Jae Young Park

Department of Urology, Korea University Ansan Hospital, Korea University College of Medicine, 123 Jeokgeum-ro, Danwon-gu, Ansan 15355, Korea TEL: +82-31-412-5190, FAX: +82-31-412-5194, E-mail: jaeyoungpark@korea.ac.kr

ORCID: https://orcid.org/0000-0002-6664-6846 
outcomes comparable to those of the open technique [4].

In most cases of PN, renal hilar control causes warm ischemia, with its attendant potential for ischemic renal injury [5]. Moreover, LPN has the risk of lengthening the warm ischemic time, due to the technical difficulty of this technique. Porpiglia et al. [6] reported that LPN is a technically challenging procedure, which requires a long learning curve, and over 150 procedures are required to obtain an acceptable outcome, regardless of the tumor's anatomical characteristics. Robotic PN is also emerging as a good option for small renal masses, with a relatively short learning curve and 5-year outcomes comparable to those of other surgical methods [7]. However, such robotic instruments are expensive, and not every hospital worldwide is equipped with a robot system.

Some studies on renal vascular segmentation suggested that clamping the branches of the renal artery can provide a bloodless operative field and a minimized risk of ischemic injury to most of the kidney $[8,9]$. However, to perform selective clamping (SC) in LPN, preoperative three-dimensional computed tomography (CT) is necessary. In addition, only experienced surgeons can perform the procedure because of its technical difficulty [9]. To overcome these limitations, we present our initial experience in the first 19 consecutive patients who underwent SC handassisted LPN (SC-HALPN). The aim of this article is to describe the technical aspects and early outcomes of this technique.

\section{MATERIALS AND METHODS}

\section{Patients}

SC-HALPN was offered to patients who provided informed consent for the surgery between May 2015 and April 2018. Preoperative evaluation of each patient was completed following the NCCN guidelines [1]. Preoperative axial CT images were examined; however, no attempt was made preoperatively to reconstruct the arterial anatomy by using axial imaging, nor did we obtain preoperative angiography scans in any patient. All patient characteristics and medical records were placed in an institutional review board-approved database and the Institutional Review Board of Korea University Ansan Hospital approved this study (approval number: IRB 2018AS0011).

\section{Surgical procedure}

The procedure is described in the enclosed video file (Supplementary material). The patient was placed in a $45^{\circ}$ lateral decubitus position. An upper paramedian incision $(7.5 \mathrm{~cm})$ was made, and a pneumatic sleeve (GelPort; Applied Medical, Rancho Santa Margarita, CA, USA) was used to permit inserting the surgeon's left hand to assist in dissecting the kidney or skeletonizing renal pedicle, and for the removal of the kidney. Two additional ports were inserted, including one port in the paramedian line threefinger width beneath the hand port for the laparoscope (10 $\mathrm{mm})$ and a working port in the paramedian line threefinger width beneath the camera port (12 mm) (Fig. 1). The colon was mobilized medially, and the proximal ureter was identified and retracted laterally. Gerota's fascia was incised and the renal capsule was exposed circumferentially around the mass, by using limits that were determined with intraoperative laparoscopic ultrasound, if necessary. The renal artery branches were dissected carefully with a laparoscopic peanut instrument and a hook electrode. Further dissection was performed using the laparoscopic peanut instrument to expose the segmental renal arteries for SC.

Once identified and isolated, the arterial branch that appears to be feeding the renal mass was clamped with a single-use mini-bulldog clamp (commonly used by neurosurgeons) (Vascu-Statt II; Scalan, St. Paul, MN, USA) (Fig. 2) [10]. After clamping the segmental artery while injecting indigo carmine and furosemide, the tumor was excised using
A

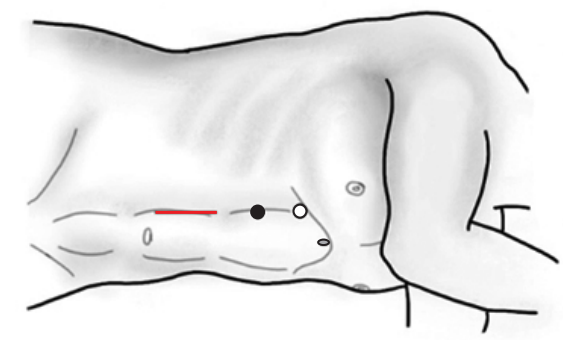

B

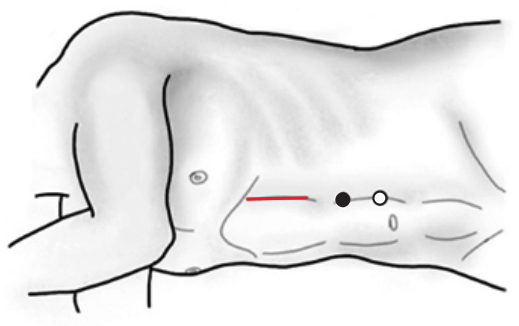

cision for hand por (along paramedian line)

- Camera port

- Port for right hand instrument

- Port for liver retraction

Fig. 1. Patients positioning and port placement. (A) Right side. (B) Left side. 

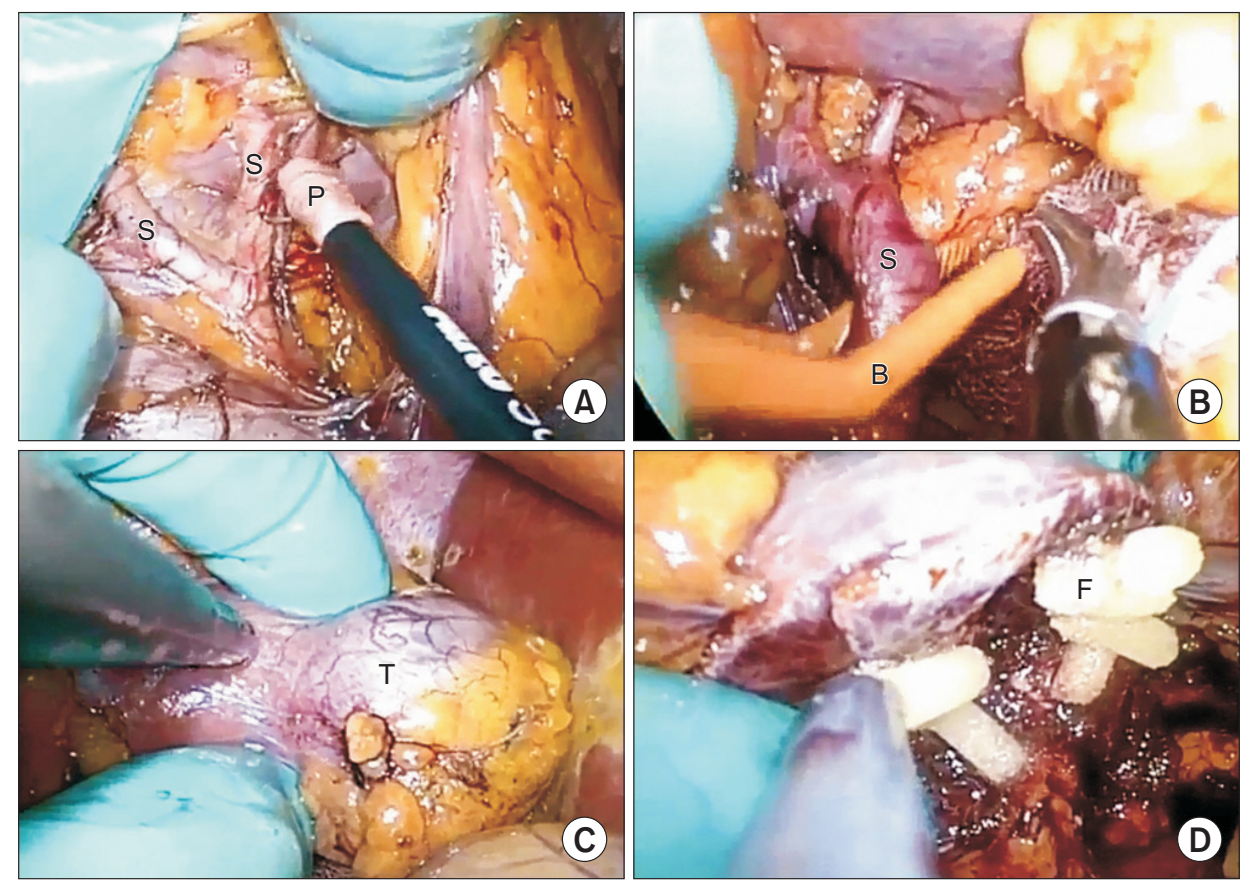

Fig. 2. Intraoperative picture of selective arterial clamping hand-assisted partial nephrectomy. (A) Segmental arteries dissection. (B) Tumor-supplying segmental artery clamped with single-use minibulldog clamps. (C) Tumor devascularization. (D) Fibrin glue on the resection bed. $\mathrm{S}$, segmental artery; $\mathrm{P}$, laparoscopic peanut dissector; $\mathrm{B}$, mini-bulldog clamps; $\mathrm{T}$, renal tumor; $F$, fibrin glue.

laparoscopic scissors to create the resection plane, and later an energy device (Sonicision; Covidien, Medtronic, Dublin, Ireland) was used to cut the tumor and achieve hemostasis simultaneously. The tumor resection bed was closed with interrupted barbed suture (3-0 V-LOC, Covidien) with Hemo-lok clips at either end. When removing the mini-bulldog clamp after suturing the resection bed, the bleeding point and urine leakage was checked (early unclamping method). After identifying the absence of urine leakage, a hemostatic matrix was applied. In case of bleeding at the resection bed, cortical renorrhaphy was carried out using cellulose bolsters secured onto a 2-0 barbed suture with Hem-o-lok clips at both ends. The drainage was removed 3 days after the operation, after confirming the absence of urine leakage through drain creatinine examination.

\section{Follow-up}

Preoperative serum creatinine was checked within 1 week before surgery, and postoperative measurements were taken within 1 week after surgery. Hemoglobin $(\mathrm{Hb})$ levels were assessed preoperatively and again on postoperative day 1. Moreover, serum creatinine and $\mathrm{Hb}$ levels were rechecked at postoperative 3 months. The estimated glomerular filtration rate (eGFR) (unit: $\mathrm{mL} / \mathrm{min}$ per $1.73 \mathrm{~m}^{2}$ ) was calculated using the modification of diet in renal disease (MDRD) equation. During the follow-up, CT urography scan was performed every $3-6$ months.

\section{Statistical analyses}

Statistical analysis was performed using Student's t-test or the Wilcoxon rank-sum test for continuous variables, and the $\chi^{2}$ test for categorical variables. All data are reported as mean and range, with $\mathrm{p}<0.05$ considered statistically significant. The cumulative sum (CUSUM) technique for the assessment of the learning curve was applied to explore the relationship between operative parameter and sequence number of the laparoscopic procedure [11]. The CUSUM series was defined as $\mathrm{Sn}=\sum\left(\mathrm{X}_{\mathrm{i}}-\mathrm{X}_{0}\right)$, where $\mathrm{X}_{\mathrm{i}}$ was an individual measurement and $\mathrm{X}_{0}$ was a predetermined reference level that was set as the mean value total operation time and estimated blood loss (EBL) for all of the cases overseen. Sn was plotted against the sequence of operations. Cutoff values were chosen according to the points of downward inflection revealed by the plots. All statistical analysis was conducted using IBM SPSS Statistics ver. 21.0 software (IBM Co., Armonk, NY, USA) and Microsoft Office Excel 2016 (Microsoft, Redmond, WA, USA).

\section{RESULTS}

\section{Patient characteristics}

SC-HALPN was attempted on 20 consecutive patients presenting with either a solid renal mass or a complex renal cyst. However, four patients who had no segmental artery branching to the tumor were switched to the conventional method. The baseline characteristics of patients, and the perioperative and pathologic characteristics of the cohort 


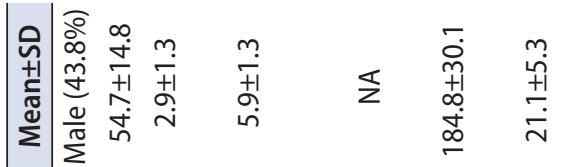

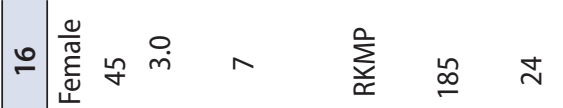

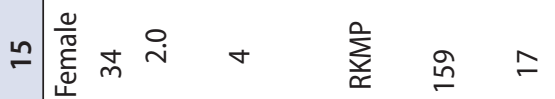

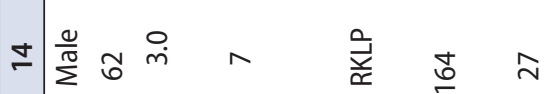

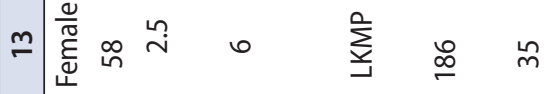

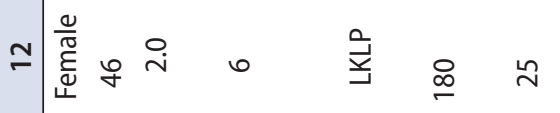

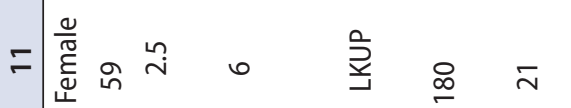

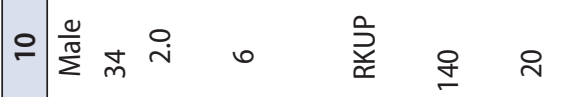

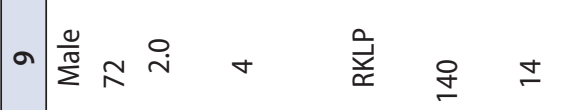

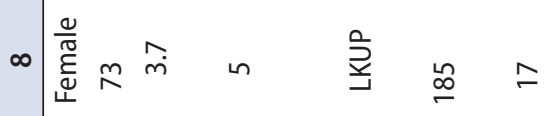

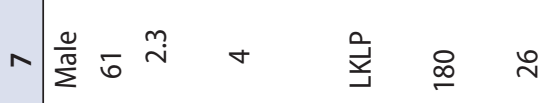

$0 \mid \frac{0}{\sum^{\pi}} n \hat{i} \quad \stackrel{a}{i} \quad \stackrel{a}{\frac{a}{N}}$

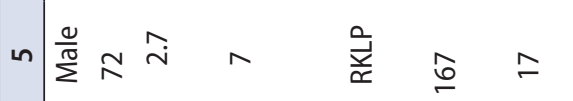

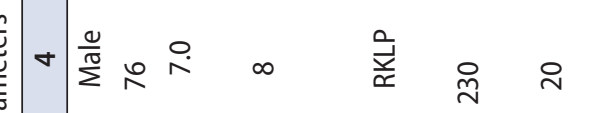

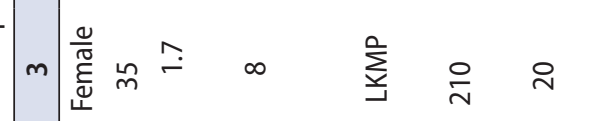

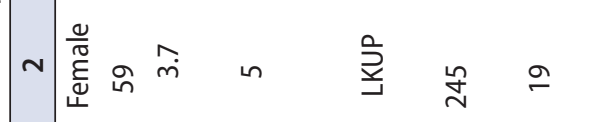

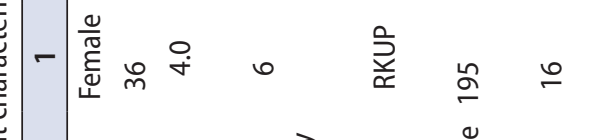

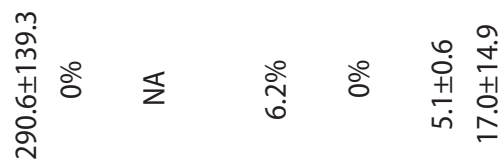

흔 응 융

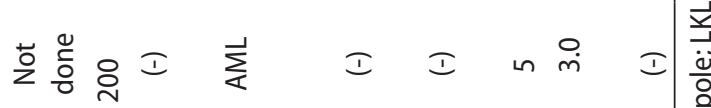

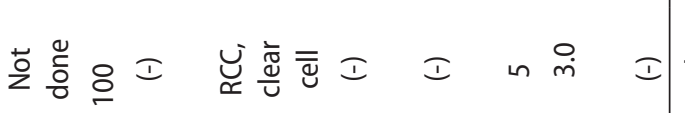

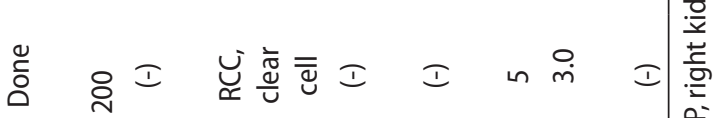

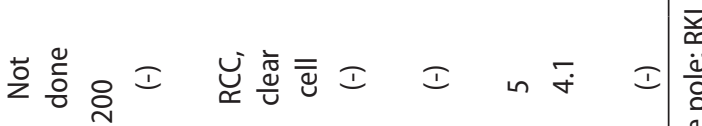

ণั๋ ঃI

윤 元 I

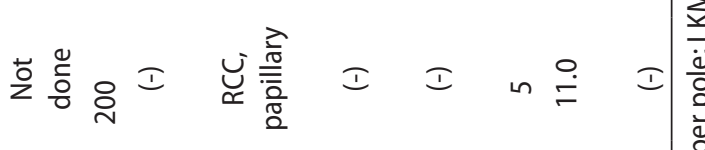

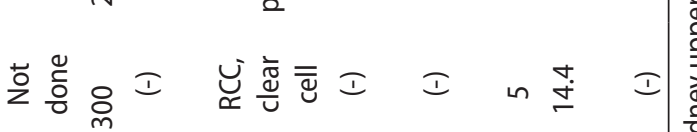

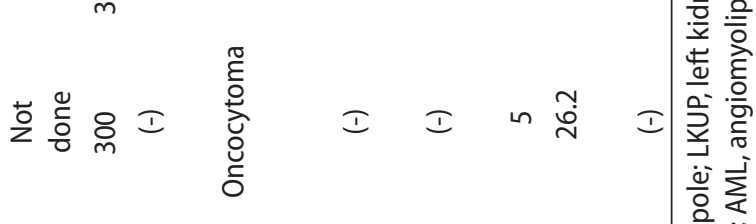

눈 융

훈 응 I

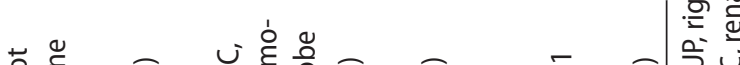

t윤

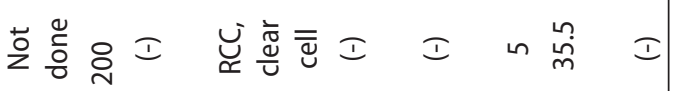

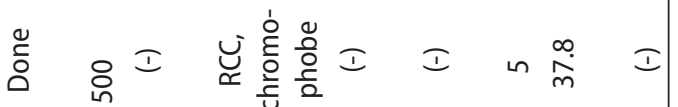

응 $\frac{\text { 음 }}{\frac{\pi}{0}}$

응 훙

䒿

है

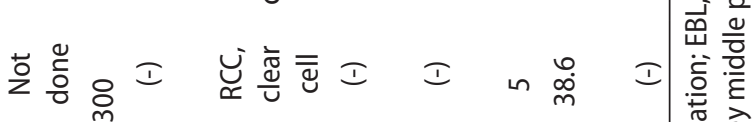


are shown in Table 1 . The mean tumor size was $2.9 \mathrm{~cm}$ (range, 1.7-7.0 cm), mean operation time was 184.8 minutes (range, 140-245 minutes), mean blood loss was $290.6 \mathrm{~mL}$ (range, 100-600 mL), and mean hospital stay was 5.1 days (range, 4-7 days). The mean selective ischemic time was 21.1 minutes (range, 16-35 minutes). No intraoperative or postoperative complications occurred, and one patient received a blood transfusion in the perioperative period. In addition, no patient had any evidence of acute or delayed renal hemorrhage, and none developed any systemic sequelae. Histopathological examination confirmed renal cell carcinoma in 13 patients (81.3\%), all with negative margins.

\section{Outcome}

The median preoperative and postoperative serum creatinine levels ( 0.91 and $0.98 \mathrm{mg} / \mathrm{dL}$, respectively) and eGFR (89.70 and $79.60 \mathrm{~mL} / \mathrm{min}$ per $1.73 \mathrm{~m}^{2}$, respectively) were comparable. The median absolute change in serum creatinine level and eGFR at postoperative 3 months were minus $0.02 \mathrm{mg} / \mathrm{dL}$ and $3.18 \mathrm{~mL} / \mathrm{min}$ per $1.73 \mathrm{~m}^{2}$, respectively
(Table 2). Among the patients, one was diagnosed as having preoperative chronic kidney disease (CKD) stage IV. The preoperative serum creatinine level and eGFR of the patient with CKD were $2.03 \mathrm{mg} / \mathrm{dL}$ and $31.1 \mathrm{~mL} / \mathrm{min}$ per $1.73 \mathrm{~m}^{2}$, respectively. However, at postoperative 3 months, the serum creatinine level and eGFR were $2.32 \mathrm{mg} / \mathrm{dL}$ and $26.0 \mathrm{~mL} / \mathrm{min}$ per $1.73 \mathrm{~m}^{2}$, respectively. To date, the patient remains under close observation without receiving dialysis. Among this cohort, seven patients showed reduction of eGFR compared with the preoperative measurement; the other patients showed no significant reduction of eGFR.

\section{Learning curve}

Fig. 3 shows a CUSUM chart of operative parameter against number of SC-HALPN procedures performed. The resulting CUSUM curve showed an increasing slope from the first to the fourth case in total operation time. After that point, the curve continuously declined after the 9th case, which meant that an acceptable level of performance was achieved. On the other hand, the 1st to 6th CUSUM

Table 2. Outcome of laboratory parameter

\begin{tabular}{|c|c|c|c|}
\hline Variable & Serum $\mathrm{Hb}$ & Serum $\mathrm{Cr}(\mathrm{mg} / \mathrm{dL})$ & $\begin{array}{c}\text { Estimated GFR } \\
\left(\mathrm{mL} / \mathrm{min} \text { per } 1.73 \mathrm{~m}^{2}\right)\end{array}$ \\
\hline Median preoperative value & $14.20(11.80-15.60)$ & $0.91(0.56-2.03)$ & $89.70(31.1-132.0)$ \\
\hline Median postoperative value & $13.20(11.10-15.00)$ & $0.98(0.57-2.01)$ & $79.60(32.0-129.0)$ \\
\hline Median absolute change at postoperative value & $0.85(-0.70-2.40)$ & $0.03(0.08-0.17)$ & $-5.90(-20.0-5.0)$ \\
\hline Median postoperative 3 mo value & $(-)$ & $0.90(0.57-2.32)$ & $89.70(26.0-165.0)$ \\
\hline Median absolute change at postoperative $3 \mathrm{mo}$ & $(-)$ & $-0.02(-0.30-0.30)$ & $3.18(-27.0-33.0)$ \\
\hline
\end{tabular}

Values are presented as median (range).

$\mathrm{Hb}$, hemoglobin; $\mathrm{Cr}$, creatinine; GFR, glomerular filtration rate.

A

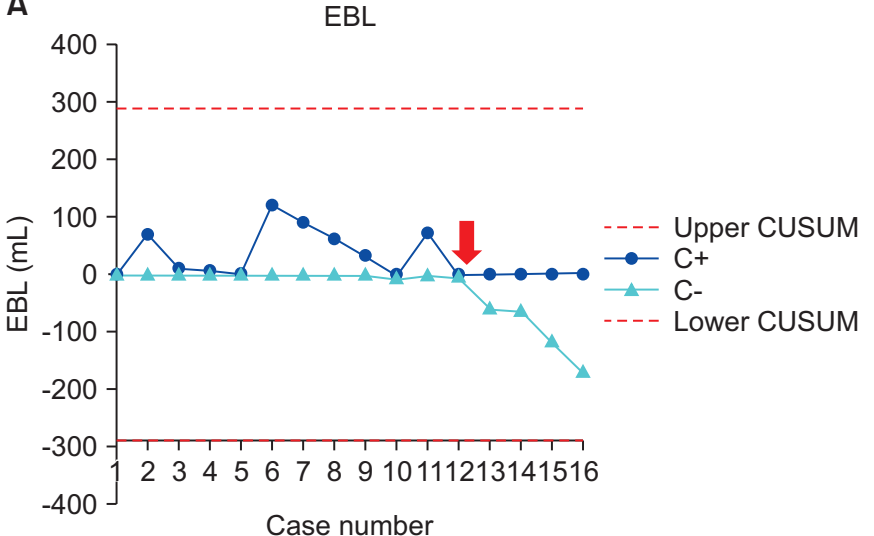

B

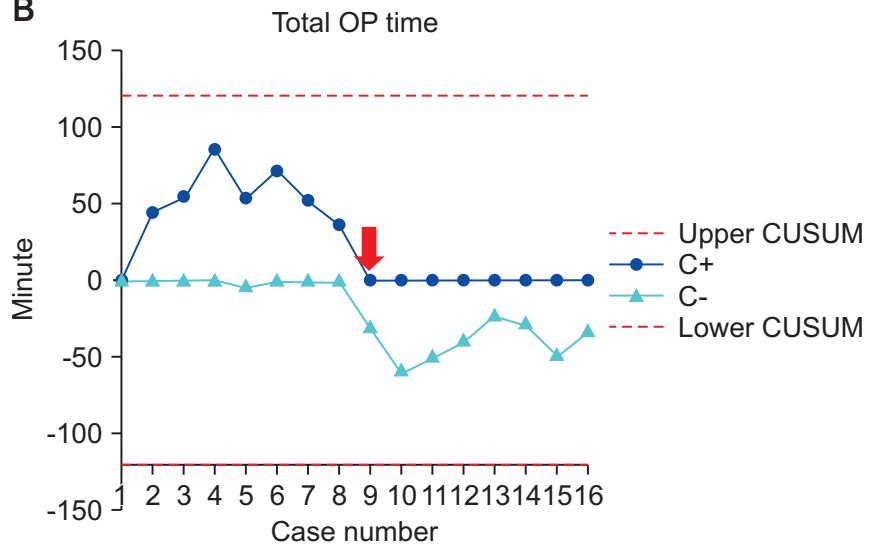

\begin{tabular}{|c|c|c|c|c|c|c|c|c|c|c|c|c|c|c|c|c|}
\hline Case & 1 & 2 & 3 & 4 & 5 & 6 & 7 & 8 & 9 & 10 & 11 & 12 & 13 & 14 & 15 & 16 \\
\hline $\begin{array}{c}\text { Tumor size } \\
(\mathrm{cm})\end{array}$ & 4.0 & 3.7 & 1.7 & 7.0 & 2.7 & 2.7 & 2.3 & 3.7 & 2.0 & 2.0 & 2.5 & 2.0 & 2.5 & 3.0 & 2.0 & 3.0 \\
\hline
\end{tabular}

Fig. 3. (A) CUSUM analysis for EBL in SC-HALPN. (B) CUSUM analysis for total operation time in SC-HALPN. CUSUM, cumulative sum; EBL, estimated blood loss; SC-HALPN, selective clamping hand-assisted laparoscopic partial nephrectomy; OP, operation. 
curves showed fluctuation in EBL. After that point, the curve continuously declined after the 12th case, which meant that an acceptable level of performance was achieved.

\section{DISCUSSION}

Although open PN has shown excellent oncologic outcomes for a long time, LPN has emerged as an alternative strategy for localized renal masses because minimally invasive surgery is the current trend. Recently, SC and off-clamping methods are emerging as attempts to reduce the warm ischemic time. The potential negative impact of warm ischemia on renal function led to the development of techniques to minimize global ischemia, such as offclamping, as described by Gill et al. [12]. They recently published a study on the unclamping anatomic zero-ischemia technique using robotic surgery and have reported that new-onset CKD stage 3 or above occurred less frequently [13]. They presented the unclamping method as a feasible procedure; however, this method is yet to be validated in other centers. Pure laparoscopic or robotic surgery is one of the most common minimally invasive procedure for patients, and its safety has been repeatedly demonstrated by previous research $[3,4,8,9,14,15]$. Nevertheless, we believe that our surgical technique can be an alternative to existing LPN or robotic PN for the reasons discussed below.

Most importantly, this surgical method does not require dedicated three-dimensional CT scan preoperatively. Most studies about minimally invasive SC or off-clamping PN reported that preoperative dedicated three-dimensional CT or magnetic resonance imaging scan with 2 to $3 \mathrm{~mm}$ cuts was required to delineate details about tumor location, depth, and proximity to the collecting system [12,16]. However, many small centers do not have a dedicated three-dimensional CT system. Especially, in developing countries, few hospitals are equipped with a dedicated three-dimensional CT system with reconstruction capabilities. In this situation, pure laparoscopy may be risky and it is difficult to expect a good surgical outcome. However, secondary-level segmental artery clamping (SAC) was safely performed without preoperative dedicated three-dimensional CT in this study. The reason for this is that tactile feedback was immediately available with the surgeon's hand. The non-requirement for preoperative CT or angiography is beneficial to the patient in terms of price and renal function preservation. Simone et al. [17] described that a dedicated renal arteriogram and superselective embolization before PN are both costly and confer the additional risk of acute tubular necrosis with contrast exposure.
In the study by Shao et al. [9], 7 of 38 cases failed and were converted to the conventional method in SC pure LPN. In patients who had variations in renal vascular anatomy and a tumor that was too close to the renal hilum, the authors reported that it was difficult to achieve clear exposure of segmental arteries and the pelvis without tumor compression. Moreover, they also reported that bleeding from a parenchymal defect could not be controlled with SAC and makes suturing difficult, requiring clamping of the main renal artery. However, we were able to expose all afferent vessels of the kidney. There were no cases in which we could not perform the procedure because of complicated vessels, except in a case with no segmental artery to the tumor region.

Secondly, SC-HALPN has a relative short learning curve compared with pure laparoscopy. LPN is technically challenging and has a steeper learning curve because it requires not only precise tumor margin resection but also a precise suture technique for the resection bed and the opened calyx [18]. In addition, the SC method is more technically challenging and difficult to implement unless the surgeon is a highly skilled expert. However, HALPN is known to be safe and easy to learn. Azawi et al. [19] presented their outcome of HALPN with early removal of the arterial clamp. The authors demonstrated that HALPN with early removal of the arterial clamp is safe and easy to perform; thus, it is believed that a laparoscopic surgeon who had performed 40 procedures can obtain a warm ischemic time of 5 minutes or less. However, the acceptable level of performance for SC-HALPN was met only in the 9th to 12th case in this study. Although SC method is technically challenging, we assumed that SC method using hand assisted laparoscopy has a relatively shorter learning curve, reflected by the rapid decrease in score difficulty and operation times.

Most of the advantages of HALPN over other laparoscopic techniques stem from the surgeon being able to insert a hand inside the patient's body during laparoscopic surgery [20]. When resecting a tumor after positioning a hilar clamp, a fingertip can bluntly dissect a mass, similar to what is achieved using a blade handle in open surgery. Moreover, using the hand to control a bleeder can be both effective and prompt. On the basis of these advantages, hand-assisted laparoscopy is a technique that ensures safe dissection of selective segmental arteries. In addition, in our experience, hand-assisted laparoscopy provided great advantages yielded by the tactile feedback. Therefore, it was much easier to find the segmental artery or accessory arteries to the tumor. 
Greene et al. [21] introduced robotic PN with retrograde renal hilar dissection and segmental arterial clamping. They described that a 'retrograde' approach to hilar dissection is a safe and feasible modification to robotic segmental clamping. It differs from our method in that hilar dissection is carried out in a retrograde fashion and using a robotic system. However, except for these two aspects, their surgical approach is the closest our approach. They reported that they did not need prototype imaging software and a dedicated CT scan to reconstruct the renal vasculature before surgery, as well as the immunofluorescence technology manufactured by Intuitive Surgical, costing nearly 150,000 USD, to assess ischemia intraoperatively [12]. The da Vinci Robotic Surgery System is available in many institutions in Korea, and is most widely used in Asia. However, even in Korea where robotic surgery is relatively popular, the da Vinci system is not easily affordable because of its cost, except for large centers. Patients may also be burdened by the approximately 8,000 USD cost of robotic PN in Korea. This may be a limitation because many developing countries do not have robotic surgery systems. However, our surgical methods have a great advantage in that they are less expensive.

It should be noted that hand-assisted laparoscopy is more invasive than pure laparoscopic or robotic PN, and can lead to wound problems and hernias. Nevertheless, in our experience, no patient had a postoperative wound problem, although this result does not have much meaning because the sample size is too small. However, Han et al. [20] reported in their retrospective study that there was only one case of postoperative hernia in 84 patients who underwent conventional PN with hand-assisted laparoscopy.

We used an energy device when excising the mass, thereby reducing blood loss during PN. However, there may be a concern that it is difficult to obtain a margin for resection by using an energy device. Bipolar devices and ultrasonic shears have the possibility of causing some degree of cellular damage, including artifact, fragmentation, and extravascular blood clotting. However, Phillips et al. [22] presented the effect of energy devices on the margin in 40 patients who underwent LPN. A bipolar device and ultrasonic shears caused some degree of cellular damage; however, these did not have a significant effect on the tumor resection margin. Rather, tumor resection by using an energy device reduced the bleeding of the renal resection bed.

In this case series, the current surgical procedure was performed by a single surgeon. The surgeon has enough experience with open PN with the SC technique and laparoscopic radical nephrectomy. However, he had no experience with pure laparoscopic SC-PN. Nevertheless, he was able to perform SC-HALPN safely and without difficulty. This demonstrates that this technique is safe and suitable for many surgeons who do not attempt performing SC-LPN because of its technical difficulty.

Graves [23] reported that the distribution of arteries within the kidney substance was constant, and thereby found the division of the renal parenchyma into five segments namely the apical, upper, middle, lower, and posterior segments. Among them, all segmental branches arise from the anterior segmental artery, except for the posterior segmental branch, which arises from the posterior segmental artery. During the transperitoneal approach, it is easy to find the branches from the anterior segmental artery because they are mostly located in front of the renal vein. Dissecting the posterior segmental branch is also not a problem because it is easily accessible when the whole kidney is dissected and rotated.

This study has some limitations. First, this is a preliminary study and the number of cases has not sufficiently accumulated to date. If many cases will be accumulated in the future, we plan to make a comparative report in a subsequent study. Second, we calculated the eGFR of patients by using the MDRD equation. However, had we calculated eGFR by using diuretic renal scintigraphy or cystatin $\mathrm{C}$ measurements, we could have more accurately reported the change in renal function.

\section{CONCLUSIONS}

SC-LPN, known to be a complicated procedure, can be safely performed using hand-assisted laparoscopic surgery with several special instruments. The procedure is promising in terms of safety and short learning curve, compared with existing surgical procedures. We introduced some tips and tricks about this procedure to aid surgeons in successfully preparing for this operation.

Currently, in South Korea, many surgeons perform robot-assisted LPN because of its minimal invasiveness and the efficacy of suturing in the process of PN using robotic arms. On the other hand, hand-assisted laparoscopic surgery has not been widely used, although it facilitates laparoscopic surgery, especially when suturing. SC-HALPN should be re-evaluated considering its cost-effectiveness, while even experienced robotic surgeons will not try to perform the SC method due to the lack of tactile feedback in the robotic system. 


\section{CONFLICTS OF INTEREST}

The authors have nothing to disclose.

\section{ACKNOWLEDGMENTS}

This research was supported by Basic Science Research Program through the National Research Foundation of Korea (NRF) funded by the Ministry of Science, ICT \& Future Planning (2017R1A2B4005876). We are also thankful to Mrs. Woo Ri Lee for providing the illustration.

\section{SUPPLEMENTARY MATERIAL}

Accompanying video can be found in the 'Urology in Motion' section of the journal homepage (http://www. icurology.org). The supplementary video clip can also be accessed by scanning a QR code, or be available on YouTube: https:/youtu.be/6GVJL4N61tM.

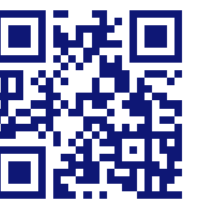

\section{REFERENCES}

1. Motzer RJ, Jonasch E, Agarwal N, Beard C, Bhayani S, Bolger GB, et al. Kidney cancer, version 3.2015. J Natl Compr Canc Netw 2015;13:151-9.

2. Herr HW. Partial nephrectomy for unilateral renal carcinoma and a normal contralateral kidney: 10-year followup. J Urol 1999;161:33-4; discussion 34-5.

3. Gill IS, Desai MM, Kaouk JH, Meraney AM, Murphy DP, Sung GT, et al. Laparoscopic partial nephrectomy for renal tumor: duplicating open surgical techniques. J Urol 2002;167:469-7; discussion 475-6.

4. Lane BR, Campbell SC, Gill IS. 10-year oncologic outcomes after laparoscopic and open partial nephrectomy. J Urol 2013;190:44-9.

5. Choi JD, Park JW, Lee SY, Jeong BC, Jeon SS, Lee HM, et al. Does prolonged warm ischemia after partial nephrectomy under pneumoperitoneum cause irreversible damage to the affected kidney? J Urol 2012;187:802-6.

6. Porpiglia F, Bertolo R, Amparore D, Fiori C. Margins, ischaemia and complications rate after laparoscopic partial nephrectomy: impact of learning curve and tumour anatomical characteristics. BJU Int 2013;112:1125-32.

7. Andrade HS, Zargar H, Caputo PA, Akca O, Kara O, Ramirez
D, et al. Five-year oncologic outcomes after transperitoneal robotic partial nephrectomy for renal cell carcinoma. Eur Urol 2016;69:1149-54.

8. Li X, Huang Y, Liu W, Li P, Tang L, Xu Y, et al. A model for assuring clamping success during laparoscopic partial nephrectomy with segmental renal artery clamping. World J Urol 2016;34:1421-7.

9. Shao P, Qin C, Yin C, Meng X, Ju X, Li J, et al. Laparoscopic partial nephrectomy with segmental renal artery clamping: technique and clinical outcomes. Eur Urol 2011;59:849-55.

10. Gill IS, Patil MB, Abreu AL, Ng C, Cai J, Berger A, et al. Zero ischemia anatomical partial nephrectomy: a novel approach. J Urol 2012;187:807-14.

11. Cundy TP, Gattas NE, White AD, Najmaldin AS. Learning curve evaluation using cumulative summation analysis-a clinical example of pediatric robot-assisted laparoscopic pyeloplasty. J Pediatr Surg 2015;50:1368-73.

12. Gill IS, Eisenberg MS, Aron M, Berger A, Ukimura O, Patil $\mathrm{MB}$, et al. "Zero ischemia" partial nephrectomy: novel laparoscopic and robotic technique. Eur Urol 2011;59:128-34.

13. Satkunasivam R, Tsai S, Syan S, Bernhard JC, de Castro Abreu AL, Chopra S, et al. Robotic unclamped "minimal-margin" partial nephrectomy: ongoing refinement of the anatomic zero-ischemia concept. Eur Urol 2015;68:705-12.

14. Furukawa J, Miyake H, Hinata N, Muramaki M, Tanaka K, Fujisawa M. Renal functional and perioperative outcomes of selective versus complete renal arterial clamping during robotassisted partial nephrectomy: early single-center experience with 39 cases. Surg Innov 2016;23:242-8.

15. Martin GL, Warner JN, Nateras RN, Andrews PE, Humphreys MR, Castle EP. Comparison of total, selective, and nonarterial clamping techniques during laparoscopic and robot-assisted partial nephrectomy. J Endourol 2012;26:152-6.

16. Simone G, Gill IS, Mottrie A, Kutikov A, Patard JJ, Alcaraz A, et al. Indications, techniques, outcomes, and limitations for minimally ischemic and off-clamp partial nephrectomy: a systematic review of the literature. Eur Urol 2015;68:632-40.

17. Simone G, Papalia R, Guaglianone S, Carpanese L, Gallucci M. Zero ischemia laparoscopic partial nephrectomy after superselective transarterial tumor embolization for tumors with moderate nephrometry score: long-term results of a single-center experience. J Endourol 2011;25:1443-6.

18. Aboumarzouk OM, Stein RJ, Eyraud R, Haber GP, Chlosta PL, Somani BK, et al. Robotic versus laparoscopic partial nephrectomy: a systematic review and meta-analysis. Eur Urol 2012;62:1023-33.

19. Azawi NH, Norus TP, Wittendorff HE, Dahl C. Hand-assisted partial nephrectomy with early arterial clamp removal: Impact of the learning curve. Scand J Urol 2014;48:538-43. 
20. Han KS, Song GH, You D, Song C, Jeong IG, Hong JH, et al. Comparison of hand-assisted laparoscopic vs robot-assisted laparoscopic vs open partial nephrectomy in patients with T1 renal masses. J Endourol 2017;31:374-9.

21. Greene RN Jr, Sutherland DE, Tausch TJ, Perez DS. Retrograde renal hilar dissection and segmental arterial clamping: a simple modification to achieve super-selective robotic partial nephrectomy. J Robot Surg 2014;8:19-22.
22. Phillips JM, Narula N, Deane LA, Box GN, Lee HJ, Ornstein DK, et al. Histological evaluation of cold versus hot cutting: clinical impact on margin status for laparoscopic partial nephrectomy. J Urol 2008;180:2348-52.

23. Graves FT. The anatomy of the intrarenal arteries and its application to segmental resection of the kidney. Br J Surg 1954;42:132-9. 